\title{
Electronic scholarly journals as information sources
}

\author{
A.M. Naven \\ Postgraduate Diploma in Information Management, Rand Afrikaans University \\ anaven@worldonline.co.za
}

\section{Contents}

1. Introduction

2. Functions of the academic journal and scholarly communication

3. What is an electronic journal?

4. Advantages of electronic journals

5. Advantages of print over electronic journals

6. Constrictive issues in the transition from print to electronic journals

7. Impact of electronic journals on information services

8. Future of electronic journals

9. Conclusion

10. References

\section{Introduction}

In 1996 Whalley (1996:1) argued that there has not been a headlong rush to publish electronically because academics still prefer to see their literature in paper form and they still prefer traditional refereeing procedures, despite its acknowledged difficulties. In the same year Harnad (1996:4) stated that serious scholars and scientist have distanced themselves from publishing their work through the electronic medium, as 'it was much too chaotic and undiscriminating a medium to be trusted with the communication and preservation of their substantive ideas and findings'.

One year later, Hobohm (1997:6) mentions that the growth of the overall number of electronic journals points to a general change of the scientific communication process. Research findings (Ashcroft 2000:467) clearly demonstrate that the number of electronic journals continues to grow. In the 18 months from September 1997 to March 1999, the number of electronic journals available increased by more than $50 \%$ to 7288 .

This article reports on an investigation into the status of the electronic journal as a medium of scholarly communication and its position as an information source. The author's opinions and conclusion is based on a literature study of published articles on the issues around electronic journals. The purpose of this theoretical discussion is to find an answer to the problem: 
How does the electronic journal affect scholarly communication and access to information?

The problem area is investigated in terms of the following issues:

- What is the purpose of academic journals in scholarly communication?

- What is an electronic journal?

- What are the advantages of electronic journals?

- What advantages do print journals have over electronic journals?

- What are the constraints in the transition from print to electronic journals?

- What is the impact of electronic journals on information services?

- What can be expected in the future regarding electronic journals?

\section{Functions of the academic journal and scholarly communication}

Before embarking on all the issues surrounding electronic journals, it might be worthwhile to look at the purpose of the academic journal in scholarly communication. Is the academic journal, in paper or electronic format, still fulfilling a need in the scientific world?

Since the first scholarly journal Journal des Scavans was published in 1665 the printed journal has been serving as the primary medium of research communication and has remained unchanged in form and function. As early as 1939 studies have shown that the volume of information being published was so vast that $75 \%$ of it was of little or no value to researchers. This might be the reason why peer reviewed journals have gained importance and are considered as 'necessary' journals. By the middle of 1995, the growth of information, particularly in scientific and technical journals, has increased tremendously and it is estimated to double every 10 to 15 years (Rao 2001:169-170).

Varian (1998:2) points out that scholarly communication also serves as an input to academic research. To improve own work and to avoid duplicating work already done, it is important to know what other researchers in the subject area are doing. Therefore, scholars generally want access to a broad range of academic journals. Scholarly communication is sharing and evaluating research results, findings and observations among professional colleagues and students by informal and formal means. Rao (2001:170) identifies two main established means of communication channels among scholars:

- Informal communication methods - personal contacts, while attending lectures, conferences, seminars, etc. This is usually called an information communication network or 'invisible college'; and

- formal communication that takes place by publishing research work in a refereed journal (printed), which is the primary channel of communication meant for a wider audience.

A scholarly journal has got certain functions in the scientific world no matter in what format it is published or distributed. Rowland (1997:1-2), Valauskas (1997:2) and Whalley

(1996:1-2) have identified the following functions:

- Communication of academic information - rapidly, accurately, widely and cheaply

- Doing more than just transfer information - it provides a vehicle for discussion - the act of publication opens a paper to commentary and criticism on a discipline-wide scale

- Dissemination of information 
- Quality control - the filtering of information is an important function provided by a high-class journal

- Preservation of the archive - it is important for posterity that the referenced and final 'lodged' material is not only archived, but also accessible to all.

A journal's status seems to be an important consideration among academics. An author's reputation and status depend on publishing in good journals, and further research funding and job promotion opportunities depend on this reputation. Recognition of authors should be seen as the most important function of academic journals (Rowland 1997:2).

Tenopir and King (1998:2) found ample evidence that scholarly journals are not only widely read, but are extremely useful and important to scientists' work, whether it be teaching, research, administration, or other activities. Furthermore, the value of the information is clearly established, whether measured by what users are willing to pay for it (purchase value) or by the benefits derived from its use (use value). In addition, information attributes (e.g. relevance, quality, accuracy) and communication attributes (e.g. availability, accessibility, ease of use, or cost to use) contribute to the use, usefulness, and value of the information. While information seeking and use patterns vary dramatically among user groups, channels of dissemination, journals, and even articles within the same journal, the usefulness and value of the information are found to be high for all of those patterns.

Despite its benefits to the academic and research community, the printed journal has been subjected to criticism from many angles such as the peer review process, delays in publication, escalating costs, lack of selectivity, stoppage of subscriptions by libraries and commercial publishers holding copyrights. In spite of non-availability, increase in volume and high price of printed journals, the academic and research community is trying to maintain research communication through newer channels like electronic journals. With the emergence of the Internet, publishing has become very easy, quick and cheap in a medium that can be accessed easily by everyone from anywhere. Print journals are rapidly moving on-line while still retaining their traditional formats. With the steady growth of e-journals on the Internet, it was found that creativity and productivity has also improved due to network technologies. Scholars have understood the power of electronic journals and seem to have accepted the new medium for communicating research ideas and results among fellow professionals. The Internet is reshaping the way in which scholars communicate with one another, changing the characteristics of scholarly communication (Rao 2001:169-171).

Hobohm (1997:7) confirms this view when he says that the new communication channels do exist and more and more scientists will want to use them. Therefore, it is imperative that transformation of journal information from traditional paper to electronic dissemination maintain or enhance information and communication attributes and continue to serve all of the diverse information-seeking and use patterns that lead to the usefulness and value of the information (Tenopir and King 1998:25).

\section{What is an electronic journal?}

Whalley (1996:2) has made a distinction between a 'pure' electronic journal, which is a journal that has been set up as a totally electronic, peer-reviewed journal, and a 'hybrid' electronic journal that has versions both in electronic and paper formats. Valauskas's (1997:1) definition leans to the idea of the 'pure' electronic journal when he defines an electronic journal as: 'a digital periodical dedicated to publishing, on the Internet, articles, essays, and analyses that have been read and commented upon initially by a select group of editors and reviewers, to meet a certain arbitrary standard of excellence (as determined by 
the editors) for a given discipline addressed by the journal itself.' Also, an electronic journal appears on a routine, although not always regular, schedule. They are formatted to have certain styles, designs and formats, just like print journals, but within the limits of the Internet medium (Valauskas 1997:1).

Ashcroft and Langdon (1999:105) defines an electronic journal as 'a journal, including indexing and abstracting services, provided by any electronic means, e.g. Internet, CDROM, although not necessarily exclusively by electronic means'. Meadows (1997:151) supports the view that electronic journals are available both online and on CD-ROM and each medium has its advantages and disadvantages. On the other hand, there is the view that electronic journals use the latest in Internet technology to disseminate ideas, which for centuries have been available only in print.

Both the pure and the hybrid types of journals may have a significant impact on scholarly communication and the way in which knowledge is created and disseminated. The electronic journal is therefore of significant interest to many of the stakeholders in the information industry, including authors, editors, publishers, subscription agents, libraries and document delivery services (Rowley 2000:46).

In view of these definitions, the author considers an electronic journal to be a serial publication that appears on a routine schedule, in any digital format, with the purpose of reporting on research activities within a specific field of expertise, whether it is peer reviewed or not. For the purpose of this research report, the focus will be placed on those electronic journals that are available online via the World-Wide Web (WWW) or Internet.

\section{Advantages of electronic journals}

Electronic journals could have certain advantages over their printed counterparts when specific features of the electronic medium are cleverly applied. Ashcroft and Langdon (1999:112) exchange the view that electronic journals offer a solution to some of the problems facing the management of the academic journal today.

One of the major advantages that are mentioned in most discussions on advantages of the electronic journal is that they enhance the speed of communication. They can reach potentially enormous academic and lay audiences in a fraction of the time and at a fraction of the cost of printed journals (Ashcroft and Langdon 1999:112). One of the main reasons of this advantage is the fact that the editorial processes occur at a faster pace than in print as a result of highly interactive communications between contributors, editors, reviewers and readers. This is facilitated by the following:

- The networking of authors, editors and reviewers. The mere electronics of digital journals makes for a more interactive and instantaneous editorial analysis. Reviewers thousands of miles apart can review an article in real time with other editors, allowing for the rapid development of comments and opinions.

- Peer review (the traditional quality control system) itself is often responsible for as much of the delay as the paper publication and distribution process itself. The electronic medium allows several ways in which the peer review process can be made more efficient, fairer and, perhaps, even more valid. It is also possible to distribute the burdens of peer review more equitably and to select referees on a broader and more systematic basis via the Internet.

The electronic medium enables a rapid production of a final product by having the means to 
avoid delays in printing. Also, there is no need to accumulate articles in order to produce a volume. Each article becomes a separate 'issue' numbered sequentially for each year (Harnad 1996:5-6; Valauskas 1997:1-3; Whalley 1996:2).

Rowland (1997:1-2) has identified another situation that the Internet has had a major positive influence, namely the potential of improving 'the invisible college' (informal channels of scientific communication), democratising and reinvigorating collaboration among scientists and radically changing academic life by almost cost-free discussion across time and space as offered by electronic mail. He says that, previously, people not situated in major research departments tended to be excluded from the invisible college. Harnad (1996:6) touches on the same principle by predicting that 'scholarly skywriting' (interactive publication in the form of open peer commentary on published and ongoing work) will prove to be the invaluable new communicative possibility offered by the Internet, the one that paper could never hope to implement. However, he sees the need that this 'scholarly skywriting' should be constrained by peer review to some extent while admitting that there is room for free (unrefereed) discussion among scholars too.

While the volume of printed material is continuously increasing at great speed, the great volume also makes it advantageous to use electronic tools to locate the material. Electronic media provide powerful searching tools that make it much easier to search for information contained in electronic journals. References can be immediately displayed using hyperlinks. Both forward and reverse bibliographic searches can be done using online materials, which should greatly aid academic writing (Ashcroft and Langdon 1999:107; Varian 1998:6).

Ashcroft and Langdon (1999:107,112), Rao (2001:172) and Varian (1998:6) refer to some other advantages of electronic journals above print journals, which are listed below:

- They are easily accessible even from an individual's desktop.

- Therefore they are saving time in waiting for information.

- It is much easier to monitor the use of electronic media.

- It enables multi-user access.

- The increase in cost for keeping printed material makes electronic forms more attractive from an economic viewpoint because of economy in storage - they are space saving.

- There are no physical barriers.

- The cost is low (sometimes free).

- Authors and readers get closer.

The value added features and services offered by electronic journals seem to be unlimited. Ashcroft and Langdon $(1999: 107,112)$ say that electronic journals can provide facilities such as integrated text, hypertext links and multimedia that the printed journal cannot offer. They are capable of supplementing traditional text with images, sound and video, opening up entirely new views of inquiry and analysis. Varian (1998:6) talks about the small incremental costs to store longer documents, making it easy to include data sets, images, detailed analyses, simulations, etc. that can improve scientific communication. Whalley (1996:2) argues that using the WWW as a distribution vehicle has got the added advantage that coloured images, which are very costly to print on paper journals, can now be included in an electronic journal without the major cost implication. Valauskas (1997:1) supports the view of Whalley when he states that an issue of an electronic journal has got the potential to be more readable and appealing to the eye than the printed format because the very electronic nature of the journal provides ample opportunities for experimentation with formats, layouts, fonts and other design features. Investigations by Daly (1998:2-9) and Baldwin (1998:2-3) have found that electronic journals are capable of offering the following services and potential benefits to the readers: 
- Access to more journals than the library may subscribe to

- Access to reader discussion groups and chat services and live comments page

- Convenience of remote access

- Electronic bookmarking

- E-mail alerts to let users know about new articles

- Full text searching capability

- Getting the latest articles sooner than they are available in the library

- Guaranteed access (no missing issues!)

- High quality colour images, often not published in printed journals due to cost

- Hypertext linking to abstracts or other articles

- Immediate access

- Improved current awareness

- Improved time management (browsing or following up on a reference when the user has a few minutes spare)

- In many subject areas, the critical aspect of having access to the backfile

- Links to multimedia, for example video clips

- Online career and job information

- Personalization: saving links to articles and searches

- Providing an article forwarding option and printer-friendly versions of articles

- Weekly table of contents via e-mail.

It is clear that electronic journals do have many advantages for scholarly communication and access to academic information. But how does this affect the paper journal?

\section{Advantages of print over electronic journals}

Despite the fact that the printed journal has been subjected to criticism from many angles such as the peer review process, delays in publication, non-availability, increase in volume and high price of printed journals, it still holds advantages over electronic media (Rao 2001:169).

Valauskas (1997:5) confirms the well-known fact that paper and computer screens work very differently. In terms of utility and convenience, information on a computer monitor cannot substitute paper. 'If there are indeed large differences in the ways in which we use paper-based information and computer-displayed information, there may indeed never be a point when electronic scholarly journals completely replace their paper counterparts. Each serves different functions for multiple audiences within a discipline.' Liew et al. (2000:302) point out that paper can hold up to 50 times more information for a given space than a monitor. One study of the use of 19000 pages of online text indicated that readers tolerated only limited amounts of electronic text, information that satisfied a specific need and did not require a great deal of scrolling through monitors.

Another study by Woodward et al. (1997:157) contradicts the belief that academics and researchers read journals at their office desk. It was found that journals are often read on trains or aeroplanes, a convenient form of background work that can be undertaken in those otherwise wasted periods of time, or at home. 'The comfort factor should also not be overlooked. When reading a printed document one can sit in a comfortable chair, shift around from time to time, and hold the paper at a convenient distance from one's eyes. The screen and the desk chair, by contrast, are in fairly fixed positions relative to each other.'

Rowley (2000:51-52) admits that there is also still some scope for technological developments. Specifically: 
- The printed word is still the best medium for reading narrative text. Most research papers are predominately text; they are difficult to read on screen.

- Colour illustrations in print are of a better quality.

- Mathematics and diacritics are better accommodated in print.

- Printed documents are portable and do not require any special equipment.

Perhaps some day, electronic journals will be as easy to read as their paper antecedents. Advances in display technology could make monitors nearly as pleasing to the eyes as print and paper. Until that happens, however, many have already begun to realize and accept that, if there are indeed large differences in the ways in which paper-based information and computer-displayed information are used, electronic journals may never be directly comparable to their print counterparts. Each serves unique functions for multiple audiences. While reading electronic journals is not the same as reading a print copy, many are beginning to acknowledge the possibility of these electronic documents offering users advanced features and novel forms of functionality beyond what is possible in print (Liew et al. 2000:302).

Rao (2001:172) is of the opinion that the inherent problems of print journals could be eliminated if authors migrate from print to the electronic medium. However, wellestablished print journals will continue to attract many scholars because of their quality and credibility. Whether or not the paradigm change will affect the whole scientific publishing process immediately and to what extent may be disputable. However, this new communication channel does exist and more and more scientists and end-users will want to use it (Liew et al. 2000:302). This shift from paper to electronic media will be greatly determined by the ability to overcome the constraints of the electronic journal, as discussed in the following section.

\section{Constrictive issues in the transition from print to electronic journals}

\subsection{Prestige of journals}

Authors consider the significance of the work they are reporting and despatch their articles to the highest-prestige journals that are likely to accept them. A high-prestige journal attracts reports of important research findings from major figures in the field. As it takes time to build up prestige, newly created electronic journals, like newly created printed journals, are therefore likely to have a low prestige rating (Meadows 1997:152). Meadows (1997:154) suggests that the easiest way of overcoming the prestige problem is to take a wellestablished, printed journal, which already has considerable prestige, and to change it into electronic form. Because electronic journals are suspected of being short-lived, many authors may hesitate to send in articles, even to a prestigious journal. The basic question, however, is whether a transmuted journal can be financially viable.

\subsection{Permanence and visibility}

Authors may not be convinced that their articles will be available for consultation in coming decades, as they are when published in equivalent paper-based journals. Overall, it seems that electronic journals may have a visibility problem, from the author's viewpoint. Authors want to publish in a journal that reaches as many of their desired target audience as possible. Access to Web-based journals is still far from total, especially for an international audience, so that printed journals may claim superiority in this respect (Meadows 1997:152).

\subsection{Speed of publication}


Since a significant part of the delay in publishing articles relates to editing and refereeing, articles contributed to electronic journals also take time to appear. However, networked communication enables the writing of joint articles, especially when the authors are in different institutions. Since the proportion of publications that are jointly written is already large, and is still growing, this is a valuable property of electronic handling and counts in favour of electronic journals (Meadows 1997:152).

\subsection{Editorial process}

In terms of the way they handle journals, many publishers are already well on the road towards full electronic publishing. This sounds positive in terms of all-electronic handling but, even in-house, many editors prefer to correct the printed version. Hence journal handling is likely to involve a mixture of electronic and paper-based material for some time to come (Meadows 1997:152-153).

\subsection{Copyright and intellectual property rights}

Since academic recognition is attributed to scholarly works published in reputed and peerreviewed journals, the authors usually give up their copyrights to the publishers in return for the publication of the article. While the electronic publishing process could result in the progressive weakening of copyright laws and intellectual ownership, commercial publishers are still unwilling to let go their grip on the costs and copyrights of scholarly information. Whether it is in the print or electronic medium, scholarly communication has to retain its scholarly content and purpose by protecting the copyright interests of both scholars and institutions (Rao 2001:170,174; Liew et al. 2000:307).

\subsection{Parallel publishing}

Publishing both the electronic and print formats of a journal may have a few advantages. It will safeguard publishers' investments and it should also help towards a smoother transition from paper-based usage to electronic handling. Storage can continue to be based on the printed version until proper arrangements for electronic storage have been worked out. Similarly, joint print and electronic distribution ensures that all the potential readers can be reached during the transition period, and gives them time to develop their facilities for handling electronic information. However, parallel publishing will not bring down the cost of journals. The main issue that needs to be considered is: 'Will not parallel publishing simply lengthen the transition period, and so compound the problems involved?' (Meadows 1997:154).

\subsection{Accessibility}

Readers want easy access. This relates back to the need for straightforward, standardized handling. At present, readers still face difficulties of access. Different journal interfaces are in use: none of them is entirely user-friendly. Speed of access might act as a major deterrent to potential readers of electronic journals, even though difficulties like networking problems are beyond the control of both publishers and other suppliers of access to electronic journals (Meadows 1997:153).

The belief that electronic journals will provide better access to journal articles is a misconception, as this statement assumes that all journal users have their own networked PC at their workplace or at home. It is certainly true that in developed countries many academics do have daily access to a networked PC, but this is not necessarily the case for students. In the less-developed countries networked PCs might be available within an institution, but they are unlikely to be widely available, and they do rely on an uninterrupted 
electricity supply! (Woodward et al.1997:157). Hobohm (1997:8-9) also mentions the fact that it will take quite a while for everyone in the world to have access to electronic journals. This relates to the famous 'last mile problem.' It may be easy to connect a whole country to new telecommunication systems but the connections for the last few meters - to reach a specific user - may be insurmountable. Certainly in developing countries this may be an important argument for upholding the print version for a certain and perhaps much longer period of time.

On a global scale, network delays are commonplace, causing the situation that access to electronic journals is not so quick and convenient. Moreover, to reach a particular full text electronic article by browsing through tables of contents one has to go through several screens. If each of these accesses is slow, the overall time to reach a required article may be quite long (Woodward et al. 1997:158).

Another aspect of accessibility is the need to use journals in a variety of locations. Portability is a valuable property, which printed journals certainly possess to a greater degree than electronic journals. This may be temporary, since more portable ways of handling electronic data are being explored. For the immediate future, however, electronic articles will usually need to be converted to paper-based form when portability is required (Meadows 1997:153).

Research results of work done by Harter and Kim (1997:12) highlight another important point, namely that a high percentage of online resources are not directly accessible from the information provided in references. 'Direct online accessibility to such resources is obviously potentially very convenient to readers, much more so than for print references. However, if readers cannot gain access to the original sources of the cited material, the references that link the citing and the cited works are much less useful than references to print sources.'

Kidd (1997:4) also mentions that the use of AdobeAcrobat PDF viewing software has not been without its difficulties. Although Acrobat software is freely downloadable, action still has to be taken to achieve this, either by individuals or by those controlling particular servers or networks. This can take time to arrange or negotiate, and acts as a barrier to some. Users may need to upgrade their current version of AdobeAcrobat on their machines to view recent issues of electronic journals. Printing from Acrobat has been another controversial area. Fairly high specification laser printers, or printers configured in particular ways, are required to print Acrobat documents reasonably speedily. Some publishers have adopted other viewing software, for example Catchword's RealPage. This software may well have some advantages over Acrobat, but requires separate downloading and registration, creating another potential barrier.

Another aspect of ease of access referred to by Kidd (1997:4) is the use of usernames and passwords. Some institutions use these on restricted Web pages only, but the majority have issued passwords individually as requested. This is a further restriction on widespread adoption of the new resource. There has been a move to control through IP registration, while retaining passwords as an alternative in certain circumstances. This provides academics and students with transparent access, and is being adopted by more publishers, certainly simplifying both administration and use.

\subsection{Archiving}

Meadows (1997:153) is of the opinion that electronic journals look good in terms of storage. They require far less space than printed journals, and retrieval from them should be easier and quicker. But there is a basic question - where will electronic journals be stored? The 
provision of appropriate storage for a large number of electronic journals itself presents difficulties. More important still is the question of long-term storage. Hardware and software continue to change rapidly with time; so stored electronic journals will need to be transferred at intervals to new configurations. The cost implications of such repeated transfers are serious. However, if responsibility for storage is left with the publisher, it raises further questions. Will the publishers (each of whom will face these same storage difficulties) really maintain and provide free access to the journals into the distant future? Will the publisher become a party to take-over agreements? How will institutional purchasers, accustomed to acquiring a material product that can be retained in-house, react to this kind of situation?

The study by Ashcroft and Langdon (1999:108) indicated that the lack of a permanent archive is seen as a considerable disadvantage. This uncertainty of continuity of material has led some institutions to exercise extreme caution before cancelling paper subscriptions. The archiving of electronic resources raises concerns about the security of data files, search software and operating systems, remote access links, and storage costs. Assuring archival access becomes an even more critical issue for particular types of products, such as electronic journals and full-text databases. Both storing large files of data and maintaining access to them present a significant additional cost in terms of staff, time and resources. Still, users are often hesitant to rely on electronic copies of titles for fear that archival access will not be maintained.

Varian (1998:5) warns that electronic archiving is not free and can, in fact, be very costly. Running a Web server or creating a CD is costly. Even more costly is updating the media. Books that are hundreds of years old can easily be read today. Floppy disks that are ten years old may be unreadable due to obsolete storage media or formatting. Electronic archives will need to be backed up, transported to new media and translated. All of these activities are costly. However, once fully implemented, electronic documents will undoubtedly reduce many of the traditional library costs.

\subsection{Cost of publishing electronic journals}

The cost of an electronic journal does not just relate to the price of the journal itself, but also to the supporting IT infrastructure. The cost of an electronic journal is usually complicated to work out (at least in contrast with a printed journal) as it will usually depend upon more variables. Currently most electronic journal subscription models are tied to a print subscription with a surcharge (Ashcroft and Langdon 1999:110).

Rowley (2000:51) reasons that the key issue in the speed of acceptance of e-journals as a substitute for print-based journals will be price. Meadows (1997:152) maintains that the matter of most concern to publishers is the financial viability of their journals. The immediate problem relates to how electronic journals should be priced, and this is unlikely to be resolved until the readership of electronic journals has been established. The many challenges of pricing electronic information have led to two types of pricing structures, as recognized by Rowley (2000:52):

- Subscription arrangements that often cover both the print and electronic versions of journals. Searching and browsing, and sometimes downloading options are included in the subscription.

- On-demand payment, or pay-as-you-view services that are designed to encourage individual users to access and use articles that match their interest profile. Pricing structures may depend on the format of the article that is viewed, and whether print versions (including facsimile) of the article are also requested. 


\subsection{Site licences}

Ashcroft and Langdon (1999:111) have found that complicated site licence arrangements pose as a significant barrier to accessing electronic journals. 'Contracts are being adapted as the vehicles for new relationships and new uses. Publishers are contracting with aggregators; users are contracting together as consortia, and consortia and publishers are forming new relationships.' Libraries are continually looking for methods to reduce the unit cost for each journal or article required by their customers. This is achieved to a great extent through purchasing as part of a consortium.

\subsection{Key concerns}

Apart from the issues discussed above, Rowley (2000:51-52) has identified key concerns that will have an impact on the transition from print to electronic journals:

- Establishment of originality - authors must submit new work that does not overlap significantly with previous submissions and must link their contribution to related contributions.

- Publisher commercial viability - through publication, journals and authors acquire standing and prestige, and publishers accumulate profits. These profits must be sufficient to support continued business success.

- Publisher competencies - publishers need to develop new skills in multimedia material, and facilitate interaction between scholars. In addition, publishers will need to acquire a much more in-depth knowledge of copyright and licensing opportunities, especially in relation to an international arena.

- Peer review - the process of peer review needs to be evaluated, in order to accommodate methodologies for reviewing and authenticating multimedia material. Reviewing multimedia material, which creates an experience, involves revisiting the objectives of scholarly communication and establishing new criteria for evaluating the effectiveness of specific documents.

- Authors' rewards and attitudes - academic staff, and authors in particular, need to relinquish the traditions of research publishing associated with print journals, which are intimately involved with tenure, promotion and grant of research moneys.

- Universities and libraries - institutions need to be convinced that electronic journals are cost-effective, and can be networked for multiple user access at a price that is acceptable to them. In particular, at the present time, electronic journals are often in a trial phase and the librarian has maintained the print subscription for archival purposes.

\section{The impact of electronic journals on information services}

\subsection{Awareness and promotion of electronic journals}

With the substantial increase in the number of electronic journals becoming available (50\% according to Ashcroft [2000:467]) in potentially different electronic formats, users need to be aware of those electronic journals in order to make full use of electronic journals. Hence promotion plays an important role. Promotion of electronic journals implies that the usefulness of materials needs to be evaluated and the material must be accessible in the variety of delivery formats available. If students, researchers and academic staff are to make full use of electronic journals, then they need to be aware not only of those electronic journals which are available but also how to access the material they require, whether in electronic format or from physical holdings (Ashcroft 2000:469). 


\subsection{Access to electronic information}

A study by Tenopir and King (1998:7) indicated that scientists obtain their articles through personal subscriptions, nearby libraries and other sources such as shared office collections, colleagues and authors. Rao (2001:172) predicts that many academics may stop going to libraries, seeing that they now have access to e-journals from their desktops. Woodward et al. (1997:159), however, say that the fact that people do not go in person to a library building does not mean that the library and other information services are now without value. These information services are doing a great deal to facilitate users' access to electronic information by means of CD-ROM networks, WWW pages providing links to national subject databases and datasets, and the development of Web-based OPACs to provide a seamless method of information retrieval. In addition to facilitating and enhancing access to electronic information, libraries have another very important role to play in the electronic publishing scenario. While free Internet journals can be accessed by anyone without a library's intervention, subscription-based journals will need a focus in each institution to deal with aspects of selection, acquisition, payment of subscriptions, issuing of passwords, etc.

Unless the prices of electronic journals are very much less than those of printed journals, individuals will still not be able to purchase all they need and, as at present, will turn to information services. Equally, tracking down electronic information and manipulating computer interfaces are complex tasks. They will require more library support than has been necessary for printed journals. Libraries will therefore continue to play a role in the circulation of journals but, as with publishers, their role will undergo some change (Meadows 1997:153).

The usefulness of dissemination of information via institutions such as libraries lies in their 'performing a valuable service to academic and research colleagues by facilitating quick and easy access to information'. Most providers offer a choice of delivery methods with the full text image (pdf file or similar) being the most popular (Ashcroft and Langdon 1999:108109). In 1997 Meadows (1997:153) pointed out that one of the basic requirements that libraries had of publishers was the need for standardized handling of electronic journals. Even now, four years later, there is still no generally accepted mode of presentation of online journals.

It is suggested that, although currently electronic journals may be a more complicated component than other materials, they have their place in collections. It should be kept in mind that collection managers should focus on the content of the scholarly information provided, regardless of the actual form in which the information arrives, and librarians should apply the same set of selection criteria to electronic journals as they apply to paper journals. Factors in selection should include high quality publication, long life expectancy and titles that are peer reviewed. Therefore, electronic journals should form part of an institution's information collection when they meet the requirements of the institution and when they meet users' needs. These factors are more important than the format of the material (Ashcroft and Langdon 1999:106).

One important role of libraries is to ensure that the best information possible is provided to their user communities at a minimum cost to users and their organizations. That rarely means one single approach to dissemination, because the wide variety of informationseeking and use patterns requires a range of dissemination mechanisms. Paper may be best for individuals who read a journal extensively and are not near a shared collection, for library and unit collections of current periodicals available to those who are located nearby, and for centralized older collections that are not available electronically. Electronic access may be best for low-circulation journals; journals that are infrequently read by individuals; 
journals that are collectively infrequently read in an organization, and older articles from journals that are discarded because they are available electronically. Such an approach is based on minimizing the cost of each situation (Tenopir and King 1998:26).

\subsection{Journal subscriptions}

Even so, according to Rao (2001:171), several studies have shown that academic and research libraries subscribe to fewer journals, despite new journals being launched every year. Apart from the problems relating to information overflow, commercial publishers have been responsible for the serials crisis. Academic institutions are concerned about the longterm impact on academic programmes unless a reasonable library resource base is maintained by providing a mix of both print and electronic journals. Libraries are pressed with the burden of maintaining the minimum number of journal titles in print at the same time as trying to provide means of access to electronic journals without additional or with little financial resources. With the advent of the Internet, more and more journals are being published in electronic media, thus enabling publishers to have stricter controls over copyrights. One thing has become very clear - that 'supply-based' information services adopted by traditional libraries are no longer viable and only 'demand-based' initiatives can be sustained in the long run. Rowley (2000:53) also talks about this problem, when he blames the changes in the economics of journal acquisitions for the shift in library strategies from holdings to access-based policies. This has led to a greater demand for channels of accessing information, including the electronic document delivery services and electronic journals. The fact is that one of the major interests of libraries is to find ways of reducing journal expenditure. If accessing electronic journals is no cheaper than buying printed journals, their enthusiasm for them is likely to be limited (Meadows 1997:153). One of the ways by which journal expenditure can be reduced is by forming groups or consortia between libraries and other institutions that enable them to subscribe to electronic journals at reduced rates (Rao 2001:173).

\subsection{Archiving of electronic journals}

Another problem that causes great concern for information services, is that of keeping archival copies of e-journals and adding them into the catalogues and holdings (Rao 2001:173). Hobohm (1997:9) agrees that unless the problem of archiving electronic publications in general will be solved, libraries have to be aware of their crucial archival role in scientific communication. Either they continue to subscribe to small journals (which are often not so expensive) or they will invent some procedure to archive electronic texts in order to keep them accessible well into the distant future.

Keeping all these problems in mind regarding the impact of the electronic journal on information services and dissemination of information in general, Rao (2001:171) predicts that the print journal is likely to remain the primary channel of formal communication in this decade, but at the same time e-journals are likely to become an increasingly important parallel source of scholarly information.

\section{Future of electronic journals}

Whether it is in the print or electronic medium, scholarly communication has to retain its scholarly content and purpose by adhering to the following principles (Rao 2001:174):

- Provide tools to help the communication process

- Allow scholars to communicate effectively and timeously 
- Eliminate barriers and limitations

- Protect the copyright interests of both scholars and institutions

- Maintain quality by peer reviews and referee systems

- Reduce costs

- Avoid delays in publishing and delivery.

It is necessary, according to Rao (2001:174) that every player has to satisfy his/her interests and at the same time continue to promote scholarly communication in spite of technological developments, growth of knowledge, changing user needs, and changing means and modes of information transfer. The Internet offers unlimited prospects for publication and distribution of information in digital form. Authors, being the prime producers of knowledge, seem to have no choice but to accept the new means of electronic publishing through the Internet in e-journals. Publishing in e-journals is gaining recognition and momentum with the inclusion of the referee system and peer reviews by major publishers. Academic institutions are beginning to accept electronic publications by their faculty for tenure and promotion purposes. Libraries have to work out strategies and actively collaborate with publishers to satisfy the needs of researchers and promote scholarly communication. Libraries, while retaining limited print-based services, have to migrate towards electronic media so that the traditional library model does not become an obstacle to the scholarly communication process in the new digital environment. To achieve a 'virtual information system' for scholarly communication, certain key issues need to be considered, such as editorial standards, high cost of retrospective conversion, database maintenance, user acceptance, pricing and copyright, archiving and reliable storage and retrieval mechanisms. Although publishers have gained supremacy over the technology, two fundamental issues need to be addressed by them, namely copyright issues and the cost of information access. They must bring in reforms with respect to electronic media so that all parties can benefit.

Liew et al. (2000:308) report on research results indicating that e-journals can be further enhanced through a more effective information environment that integrates various novel, useful tools and features to support advanced interactions between users and the various information objects contained within the e-documents.

The ultimate goal remains to provide users with access to information that will contribute to their work. Hence, according to Liew et al. (2000:308), future e-journals are likely to be characterized by the following:

- Homogeneity. Future e-journals will not be a single physical entity that contains a finite amount of static, linear content. They will not be organized and stored by arrangements that might limit the creative ability of users in browsing, searching and, eventually, synthesizing, diverse contents.

- Related and interconnected. Ideally, there would be an environment that fully integrates access to heterogeneous items. Description of the linked items as documents would need to encompass navigation paths to sites.

- Contextual display/mapping of linked items. With advancement in user interface and display technologies such as information visualization technologies, sets of related items could be ranked by, for instance, subject similarity and be displayed using twodimensional or three-dimensional technologies to help visualize ranked sets of items. This would further help users in browsing as well as directed searching.

- Malleable items. In future e-journals, users will be able to link and manipulate documents or parts of documents into sets of related ideas. E-journal collections and the information objects (e.g. a single collection, an article, a paragraph, a sentence, a word) become malleable. They can be transformed indefinitely as the user perceives new relationships. 
It is evident that for e-journals to survive and thrive, they must be different from their print forerunners. Designers and publishers of e-journals must fully exploit the electronic medium's basic properties - with 'interactivity' as the primary characteristic of new technologies. With interactive features, end-users are recognized as active participants in the communication process. Users will increasingly be seeking or selecting information more than simply receiving the information contained within e-documents (Liew et al. 2000:308).

Meadows (1996:33) refers to two factors that may conceal problems yet to come. The first factor is subsidization. Acquisition of information via the Internet is currently cheap as a result of extensive subsidization of the infrastructure in the past. If network charges increase, which seems a possibility; users will be faced with unpleasant new bills. For libraries, there is also the question of how costs of handling electronic journals compare with printed journals. There are necessarily set-up costs in making arrangements for a journal to be acquired in electronic form from a publisher. These will be considerably increased if different publishers make their journal titles available in different ways. With publishers' current anxieties about copyright protection, this generates its own difficulties. Any reasonable estimate suggests that the cost to an institution, in terms of money and effort, of introducing new electronic journals is liable to be considerably higher than for new printed journals. The second factor is standardization. Meadows (1996:33) mentions that standardization of software is still awaited. Graphics provision over networks is not all it should be and international dissemination, in particular, can be subject to irritating delays.

In this dynamic concept of electronic documents, content is in the control of end-users. The ability to organize and browse the conceptual spaces of e-journals and electronic articles, as well as relevant resources, would be highly desirable as these information resources develop further (Liew et al. 2000:308).

\section{Conclusion}

While maintaining the scholarly content, e-journals seem to have provided a great relief to the academic and research community. However, according to Rao (2001:172) the present shift in this direction is yet to satisfy the most important aspects of academic research, namely ownership of ideas, societal recognition, authenticity and claiming priority over a discovery.

It is widely expected that a great deal of scholarly communication will move to an electronic format. The Internet offers much lower cost of reproduction and distribution than print, the scholarly community has excellent connectivity and the current system of journal pricing seems to be too expensive. Varian (1998:1) states that each of these factors is helping to push journals from paper to electronic media. However, the author agrees with Rao (2001:171) that the print journal is likely to remain as the primary channel of formal communication for some time to come, while e-journals are likely to become an increasingly important parallel source of scholarly information.

Therefore, it may be concluded that the status of the electronic journal as a medium of scholarly communication and its effect on access to academic information is increasing all the time. While it seems highly unlikely that the electronic journal will replace its printed counterpart completely in the near future, wise academics, researchers and information service providers will stay in touch with developments regarding electronic journals. Even though issues like reputation, quality, copyright and security still need to be settled, it has become crucial for researchers to make sure that they are aware of all the relevant electronic information sources and that these sources are consulted on a regular basis for the latest 
information in the subject area.

\section{References}

Ashcroft, L. and Langdon, C. 1999. Electronic journals and university library collections. Collection Building, 1999 18(3):105-114.

Ashcroft, L. 2000. Win-win-win: can the evaluation and promotion of electronic journals bring benefits to library suppliers, information professionals and users? Library Management, 21(9):466-471.

Baldwin, C. 1998. SuperJournal update. Ariadne, 14(March). [Online]. Available WWW: http://www.ariadne.ac.uk/issue14/superjournal/intro.html.

Daly, B. 1998. Launching an electronic magazine: an overview of value-added features and services. Ariadne, 18(1-12 December). [Online]. Available WWW: http://www.ariadne.ac.uk/issue18/Web-magazine/intro.html.

Harnad, S. 1996. Implementing peer review on the Net: scientific quality control in scholarly electronic journals. In: Peek, R. and Newby, G. (eds.) Scholarly publication: the electronic frontier. Cambridge: MIT Press. [Online]. Available WWW:

http://cogsci.soton.ac.uk/ harnad/Papers/Harnad/harnad96.peer.review.html.

Harter, S.P. and Kim, H.J. 1997. Electronic journals and scholarly communication: a citation and reference study. The Journal of Electronic Publishing, 2(2):1-16. [Online]. Available WWW: http://www.press.umich.edu/jep/archive/harter.html.

Hobohm, H. 1997. Changing the galaxy: on the transformation of a printed journal to the Internet. [Online]. Available WWW:

http://www.firstmonday.dk/issues/issue2_11/hobohm/index.html\#Going Online.

Kidd, T. 1997. Are print journals dinosaurs? Ariadne, 12(November). [Online]. Available WWW: http://www.ariadne.ac.uk/issue12/main/intro.html.

Liew, C.L., Foo, S. and Chennupati, K.R. 2000. A study of graduate student end-users' use and perception of electronic journals. Online Information Review, 24(4):302-315.

Meadows, J. 1996. Opinion paper: surveying electronic journals. Interlending and Document Supply, 24(4):32-33.

Meadows, J. 1997. Can we really see where electronic journals are going? Library Management, 18(3):151-154.

Rao, M.K. 2001. Scholarly communication and electronic journals: issues and prospects for academic and research libraries. Library Review, 50(4):169-175.

Rowland, F. 1997. Print journals: fit for the future? Ariadne, 7(1-4 January). [Online]. Available WWW: http://www.ariadne.ac.uk/issue7/fytton/intro.html.

Rowley, J. 2000. The question of electronic journals. Library Hi Tech, 18(1):46-54.

Tenopir, C. and King, D.W. 1998. Designing electronic journals with 30 years of lessons 
from print. The Journal of Electronic Publishing, 4(2). [Online]. Available WWW: http://www.press.umich.edu/jep/04-02/king.html.

Valauskas, E.J. 1997. First Monday and the evolution of electronic journals. The Journal of Electronic Publishing, 31:1-8. [Online]. Available WWW:

http://www.press.umich.edu/jep/03-01/FirstMonday.html.

Varian, H.R. 1998. The future of electronic journals. The Journal of Electronic Publishing, 4(1). [Online]. Available WWW: http://www.press.umich.edu/jep/04-01/varian.html.

Whalley, B. 1996. Electronic journals, evolutionary niches. Ariadne, 3(1-3 May). [Online]. Available WWW: http://www.ariadne.ac.uk/issue3/ggg/intro.html.

Woodward, H. et al. 1997. Electronic journals: myths and realities. Library Management, 18 (3):155-162.

\section{Disclaimer}

Articles published in SAJIM are the opinions of the authors and do not necessarily reflect the opinion of the Editor, Board, Publisher, Webmaster or the Rand Afrikaans University. The user hereby waives any claim he/she/they may have or acquire against the publisher, its suppliers, licensees and sub licensees and indemnifies all said persons from any claims, lawsuits, proceedings, costs, special, incidental, consequential or indirect damages, including damages for loss of profits, loss of business or downtime arising out of or relating to the user's use of the Website. 